\title{
Relación del desempeño académico de estudiantes de primer año de universidad en Chile y los instrumentos de selección para su ingreso
}

\author{
Vergara-Díaz, Gastón; Peredo-López, Hernán \\ Relación del desempeño académico de estudiantes de primer año de universidad en Chile y los instrumentos de \\ selección para su ingreso \\ Revista Educación, vol. 41, núm. 2, 2017 \\ Universidad de Costa Rica, Costa Rica \\ Disponible en: http://www.redalyc.org/articulo.oa?id=44051357006 \\ DOI: http://dx.doi.org/10.15517/revedu.v41i2.21514
}

Esta obra está bajo una Licencia Creative Commons Atribución-NoComercial-SinDerivar 3.0 Internacional. 


\section{Relación del desempeño académico de estudiantes de primer año de universidad en Chile y los instrumentos de selección para su ingreso}

Relationship of academic performance of students in first year of university in Chile and entry instruments for their selection

Gastón Vergara-Díaz [1]

Universidad Austral de Chile, Chile

DOI: http://dx.doi.org/10.15517/revedu.v41i2.21514

gastonvergara@uach.cl

Hernán Peredo-López [2]

Universidad de La Frontera, Chile

hperedolopez@gmail.com

Recepción: 17 Octubre 2015

Aprobación: 01 Diciembre 2016

\section{Resumen:}

Si bien el rendimiento académico del estudiantado que ingresa a las universidades chilenas ha sido objeto de múltiples estudios, pocas investigaciones han analizado la relación que tienen los instrumentos de selección de ingreso y el rendimiento académico posterior. En una muestra de 440 estudiantes de Ingeniería Comercial de la Universidad Austral de Chile, pertenecientes a cinco promociones de primer año, se estudiaron los instrumentos de admisión a la carrera, y se determinó cuáles explican mejor el rendimiento académico. Para ello se utilizaron los resultados de las pruebas de selección universitaria (PSU) y las notas de enseñanza media (NEM) obtenidos por el estudiantado, en relación con el promedio semestral ponderado (PSP) del primer año universitario. Se determinó que el promedio de NEM explica mejor el rendimiento académico estudiantil, al presentar la mejor correlación con el rendimiento académico del primer semestre y de manera más fuerte en el segundo.

Palabras Clave: Notas de enseñanza media, test admisión universidad, rendimiento académico.

\section{Abstract:}

Several studies have been carried outundertaken in relation to the academic performance of freshman students enrolled in Universities from Chile. However, few studies have focused on the relationships between the entrance selection instruments applied and their academic performance at the university. A sample of 440 students of commercial engineering belonging to five different promotions from the Universidad Austral de Chile was selected to study the instruments used for admission to this career. The instruments evaluated were the university selection test (PSU) and grade average of high school showed by the students

\section{NotAS DE AUTOR}

[1] Profesor auxiliar del Instituto de Estadística (Universidad Austral de Chile), magíster en Administración de Empresas (MBA) (Universidad Austral de Chile), Doctor(c) en Recursos Naturales y Sostenibilidad (Universidad de Córdoba, España), Diplomado en Docencia Universitaria de Calidad (Universidad Austral de Chile). Líneas de investigación declaras a Conicyt: Monitoreo del cambio de recursos naturales, estadísticas e indicadores evolutivos de recursos naturales, modelamiento biométrico. Publicaciones recientes: con Víctor Jacques et al. (2014), ANALYSIS OF THE CONVERGENCE PROCESS IN THE ACADEMIC PERFORMANCE: The Case of a Chilean University; con Concha M., et al. (2012), Intención y desarrollo de competencias en investigación clínica en programas de postítulo de médico especialista en Chile; con Villarroel P. et al. (2013), Comprensión pública de la ciencia en Chile: Adaptación de instrumentos y medición; Proyectos de investigación relevantes recientes: Coinvestigador proyectos de investigación: Fondecyt 1131099; 2013 - 2015. Comunicación pública de la ciencia y modernización reflexiva: Estudio de los fenómenos de reflexividad y ambivalencia en las actitudes públicas hacia la ciencia en Chile en el marco de conflictos y problemas ambientales; Fondecyt 1085071; 2008 - 2010. Caracterización y análisis comparado del interés, comprensión y actitudes hacia la ciencia en habitantes de tres ciudades universitarias del sur de Chile: Valdivia, Temuco y Concepción.

[2] Ingeniero Forestal, estudió en Universidad Austral de Chile, titulado en Universidad de Chile: 1967. Doctor en Ciencias Forestales, Universität zu Göttingen, 1973. Magister en Educación, mención Evaluación Educacional, Universidad de La Frontera, Temuco, Chile, 2001. Profesor titular Universidad Austral de Chile, 1966 - 2007. Actualmente Profesor Ad Honorem, Departamento de Ciencias Forestales, Universidad de La Frontera, Temuco, Chile. Publicaciones recientes: Peredo H. Formación de capital humano de calidad y pertinente con las demandas sociales, laborales y tecnológicas de la Región de Los Ríos. En Región, Universidad y Desarrollo Sostenible. 60 años UACh. Ediciones Universidad Austral de Chile (en prensa); Peredo, H. Epidemiología de Dothistroma septospora en plantaciones de Pinus radiata: de la teoría a la práctica en el sector forestal chileno. 3er Congreso Argentino de Fitopatología, Junio 2014, Tucumán, Argentina; Peredo, H. Reflexiones conceptuales y propuestas metodológicas para el diseño e implementación de un currículo pertinente y de calidad. 3er Congreso Argentino de Fitopatología, Junio 2014, Tucumán, Argentina; Peredo, H. 2013. Propuesta curricular no tradicional en su gestación, para formar Ingenieros Forestales relevantes, contextualizados y de calidad. Quebracho 21(1,2),121-131; Peredo, H. 2009. Las enfermedades emergentes en el sector agrícola-forestal mundial, ¿̇requieren una re contextualización en la enseñanza de la patología vegetal? XIII. Jornadas Fitosanitarias Argentinas, Santiago del Estero; Peredo, H. 2007. Demandas sociales y disciplinares indispensables en la actualidad, para la formación de Ingenieros Forestales relevantes y de calidad. En F. Drake (Ed.), Educación Forestal en América Latina. Universidad de Concepción - FAO: 174 - 185 
in relation to the grade point average (GPA) of the first year at the University. The grade average of high school explains better the academic performance of the students in comparison to the PSU, showing a higher correlation for the first and second academic semester.

KEYWORDS: Grade average of high school, college admission test, academic performance.

\section{INTRODUCCIÓN}

La preocupación por las pruebas de ingreso a la universidad es una tendencia mundial creciente y sus efectos se analizan con base en estudios de seguimiento que permiten discriminar respecto a la mejor capacidad predictiva del desempeño académico estudiantil en la universidad (Edwards, Coates y Friedman, 2012). En Chile, durante 35 años se aplicó la Prueba de Aptitud Académica (PAA) como sistema de ingreso a las universidades, en conjunto con las notas de enseñanza media (NEM), así se otorgaba un puntaje de ingreso a la educación universitaria para cada estudiante. Los cambios en la malla curricular de las enseñanzas básica y media llevaron hace una década al Consejo de Rectores de las Universidades Chilenas (CRUCh) a elaborar un nuevo instrumento de ingreso, conocido como Prueba de selección universitaria (PSU), la cual evalúa tanto las habilidades intelectuales como los modos de operación y métodos generales aplicados a la resolución de problemas en las pruebas de lenguaje y comunicación, matemática, historia y ciencias sociales, y ciencias, esta última abarca biología, física y química. Los resultados de este cambio no han sido los esperados, incluso han perjudicado a los grupos vulnerables que ingresan a la universidad (Koljatic y Silva, 2010) y la PSU presenta además una leve subvaloración de las mujeres en la PSUM, que se compensan con una leve sobrevaloración de género al considerar conjuntamente las NEM (Manzi et al., 2010).

Frente a estas evidencias, varias universidades chilenas iniciaron, desde hace algunos años, programas complementarios de selección de estudiantes de mejor rendimiento durante la enseñanza media (Koljatic y Silva, 2013) y en la actualidad este tipo de programa está implementado en unas 17 universidades chilenas, sin que ninguno de ellos se haya formalizado a nivel ministerial (Gil, Paredes y Sánchez, 2013).

Algunos estudios nacionales señalan la importancia de las NEM como factor preponderante en la predicción del rendimiento académico, en Ingeniería Forestal (Giusti, 2004), y Tecnología Médica (Rocha, Acebedo y Flores, 2009); sin embargo, este factor puede ver disminuida su capacidad, por el manejo discrecional de las ponderaciones que hacen anualmente las universidades del CRUCh.

La búsqueda de mejores predictores de rendimiento académico ha llevado a considerar, además de las NEM, el ranqueo del alumnado en sus respectivos colegios, lo que ha mostrado buenos resultados generales en universidades chilenas (Gil et al., 2013), la misma tendencia se constata en tres universidades específicas (Meneses, Parra y Zenteno, 2005) y también se ha podido establecer una mejora de las notas en primer año de la carrera de Derecho (Meneses y Toro, 2012). La evaluación externa del desempeño de la PSU hasta el año 2012, muestra que el ranqueo de estudiantes, las NEM y las PSU Matemática y Ciencias, tienen mejor calidad predictiva del desempeño académico que la PSU (Pearson, 2013).

Considerando entre otros, estos antecedentes, el Consejo de Rectores de las Universidades Chilenas (CRUCh) decidió hacer una modificación en el proceso de admisión a las universidades chilenas para el año 2014, incorporando un ranqueo de notas de enseñanza secundaria con una ponderación del 10\%, que se reduce en el puntaje PSU y hace pública además la descripción del ranqueo, dada la repercusión social de la medida (Departamento de Evaluación, Medición y Registro Educacional [DEMRE], 2013). Se postula incluso que aumentando el peso del ranqueo de notas de enseñanza media a $20 \%$, la validez predictiva del desempeño académico de la PSU se hace menos significativa y el ranqueo de las NEM mejora su predictividad (Larroucau, Ríos y Mizala, 2013).

El objetivo del presente estudio es aportar mayores antecedentes acerca de los criterios de selección para el ingreso a la universidad que predigan mejor el rendimiento académico universitario del estudiante, centrando el análisis en las notas de enseñanza media, y los puntajes PSU. Nos hacemos partícipes, de esta forma, de 
una de las principales propuestas de la consultora Pearson (2013): investigar todos los aspectos que puedan mejorar el proceso de admisión a las universidades chilenas y la predicción del desempeño de estudiantes en la universidad.

\section{Metodología}

Los datos corresponden a una muestra de estudiantes de primer año, que incluye cinco promociones completas de la Carrera de Ingeniería Comercial de la Universidad Austral de Chile, con un total de 440 estudiantes, que se distribuyen en $42 \%$ mujeres $(n=185)$ y $58 \%$ hombres $(n=255)$, con edades entre 18 y 20 años $(M=18.4$ y D.E $=1.23)$.

Respecto al origen geográfico del estudiantado, el 72\% ( $\mathrm{n}=317)$ proviene de la Región de los Ríos, el 17\% $(\mathrm{n}=74)$ de la Región de los Lagos y el 11\% $(\mathrm{n}=48)$ proviene de otras regiones del país.

En relación con el tipo de establecimiento de origen ${ }^{[3]}$, el 42.07\% $(n=185)$ egresó de establecimientos de dependencia municipal, el 39.52\% $(n=174)$ de establecimientos particulares subvencionados y el $18.40 \%$ $(\mathrm{n}=81)$ de colegios particulares pagados.

La principal fuente de datos correspondió a los obtenidos de los sistemas de admisión y matrícula de la universidad, estos son: prueba de selección universitaria, notas de enseñanza media, colegio de origen y zona geográfica de procedencia. Los datos relacionados con el rendimiento académico de estudiantes son: promedio semestral ponderado del primer y segundo semestre y promedio general ponderado (PGP), se obtuvieron de las bases de datos del Departamento de Registros Académico Estudiantil.

Para el procesamiento de la información, se prepararon bases de datos de todo el estudiantado en cada una de las promociones, se ordenó la información con los antecedentes de ingreso a la universidad y el rendimiento académico obtenido durante el primer año académico.

El análisis de los datos se realizó utilizando el programa estadístico de acceso libre $\mathrm{R}$ (versión 3.0.3 R Development Core Team, Viena, Austria) y la aplicación de procedimientos estadísticos, correspondió específicamente a estadísticos descriptivos, comparación de poblaciones e identificación de patrones de correlación. Para la comparación de grupos se utilizó el test paramétrico para dos muestras independientes Tstudent, previo análisis del cumplimiento de supuestos de aplicación y para el análisis correlacional se aplicó el estadístico de correlaciones simples, producto momento de Pearson.

\section{ANÁLISIS Y DISCUSIÓN DE RESULTADOS}

El estudiantado de las cinco cohortes estudiadas que ingresó a primer año de la carrera de Ingeniería Comercial obtuvo un promedio de notas de enseñanza media (NEM) entre 5,0 y 6,9, con una media de 5,90 (D.E = 0,37). El mayor puntaje PSU se presentó en Matemáticas con 800 puntos $(\mathrm{M}=594,95 ; \mathrm{D} . \mathrm{E}=49,94)$ y el mínimo correspondió a Ciencias con $342(\mathrm{M}=535,74$; D.E=56,39) (tabla 1). 
TABLA 1

Estadisticos descriptivos por sexo del rendimiento académico en la enseñanza media y factores de ingreso de los estudiantes de Ingeniería Comercial

\begin{tabular}{|c|c|c|c|c|c|c|}
\hline & \multicolumn{3}{|c|}{ Hombres } & \multicolumn{3}{|c|}{ Mujeres } \\
\hline & Media & D.E. & $\mathrm{N}$ & Media & D.E. & $\mathrm{N}$ \\
\hline Puntaje PSU Matemáticas & 603.40 & 50.22 & 255 & 583.31 & 47.25 & 185 \\
\hline Puntaje PSU Lenguaje & 580.25 & 60.86 & 255 & 566.08 & 57.68 & 185 \\
\hline Puntaje PSU Historia & 599.15 & 74.96 & 191 & 559.25 & 72.18 & 140 \\
\hline Puntaje PSU Ciencias & 542.49 & 60.07 & 146 & 527.01 & 50.16 & 113 \\
\hline Puntaje Promedio PSU & 592.16 & 39.17 & 255 & 588.61 & 39.50 & 185 \\
\hline $\begin{array}{l}\text { Promedio de notas } \\
\text { Enseñanza media }\end{array}$ & 5.83 & 0.38 & 255 & 6.00 & 0.35 & 185 \\
\hline
\end{tabular}

Nota: Elaboración propia.

Con el objetivo de comprobar la existencia de diferencia significativas de género a través de las diferentes puntajes de PSU y la NEM, se aplicó el test estadístico T-student para muestras independientes con contrastes bilaterales, encontrándose diferencias significativas en las NEM $(\mathrm{p}<0,01)$ y todas los puntajes de PSU $(\mathrm{p}<0,01 ; \mathrm{p}<0,05)$, (tabla 2).

TABLA 2

Comparación NEM y PSU para mujeres y hombres

\begin{tabular}{|c|c|c|c|c|c|c|}
\hline & Sexo & $\mathrm{N}$ & Media & D.E. & Levene & $\mathrm{T}$ \\
\hline \multirow{2}{*}{$\begin{array}{l}\text { Promedio de notas de en- } \\
\text { señanza media }\end{array}$} & Mujer & 185 & 6,00 & 0,35 & \multirow{2}{*}{$1,98 \mathrm{~ns}$} & \multirow{2}{*}{$4,66(* *)$} \\
\hline & Hombre & 255 & 5,83 & 0,38 & & \\
\hline \multirow{2}{*}{ Puntaje PSU Lenguaje } & Mujer & 185 & 566,08 & 57,68 & \multirow{2}{*}{$0,49 \mathrm{~ns}$} & \multirow{2}{*}{$2,47(* *)$} \\
\hline & Hombre & 255 & 580,25 & 60,86 & & \\
\hline \multirow{2}{*}{$\begin{array}{l}\text { Puntaje PSU Matemáti- } \\
\text { cas }\end{array}$} & Mujer & 185 & 583,31 & 47,25 & \multirow{2}{*}{$0,31 \mathrm{~ns}$} & \multirow{2}{*}{$4,25(* *)$} \\
\hline & Hombre & 255 & 603,40 & 50,22 & & \\
\hline \multirow[t]{2}{*}{ Puntaje PSU Historia } & Mujer & 140 & 559,25 & 72,18 & \multirow{2}{*}{$0,42 \mathrm{~ns}$} & \multirow{2}{*}{$4,86\left(^{* *}\right)$} \\
\hline & Hombre & 191 & 599,15 & 74,96 & & \\
\hline \multirow[t]{2}{*}{ Puntaje PSU Ciencias } & Mujer & 113 & 527,01 & 50,16 & \multirow{2}{*}{$1,86 \mathrm{~ns}$} & \multirow{2}{*}{$2,21(* *)$} \\
\hline & Hombre & 146 & 542,49 & 60,07 & & \\
\hline
\end{tabular}

${ }^{*} \mathrm{p}<0,05 ;{ }^{* *} \mathrm{p}<0,01 ;$ ns: no significativo

Nota: Elaboración propia.

En el caso de nuestra investigación, considerando el primer y segundo semestre universitario, la totalidad del alumnado estudiado en las cinco cohortes mostró una mayor relación directa significativa entre el promedio de notas primer semestre universitario y las NEM $(\mathrm{r}=0,321 ; \mathrm{p}<0,01)$, como así también con el puntaje PSU de Matemáticas $(\mathrm{r}=0,356 ; \mathrm{p}<0,01)$; existen, además, relaciones significativas menores del rendimiento universitario con los demás puntajes de PSU. En el segundo semestre universitario, el rendimiento académico promedio se relaciona también, de manera positiva, con la NEM; pero en un grado mayor $(\mathrm{r}=0,347, \mathrm{p}<0,01)$ y se produce una menor correlación significativa en las diferentes PSU, con excepción de Historia, la cual pasó a ser no significativa (tabla 3, figura 1). 
TABLA 3

Correlación del rendimiento académico en la enseñanza media y factores de ingreso en el rendimiento académico del primer año universitario

\begin{tabular}{|c|c|c|}
\hline & Promedio primer semestre & Promedio segundo semestre \\
\hline Promedio notas enseñanza media & $0,321(* *)$ & $0,347(* *)$ \\
\hline Puntaje PSU Lenguaje & $0,272(* *)$ & $0,168{\left({ }^{* *}\right)}^{*}$ \\
\hline Puntaje PSU Matemáticas & $0,356\left(^{(* *)}\right.$ & 0,160 (**) $^{*}$ \\
\hline Puntaje PSU Historia & $0,166($ (**) & 0,067 \\
\hline Puntaje PSU Ciencias & $0,295(* *)$ & $0,157\left(^{*}\right)$ \\
\hline
\end{tabular}

Nota: Elaboración propia.

El estudio de Bravo et at. (2010) plantea que las notas de enseñanza media (NEM) mantienen una capacidad predictiva alta para todos los años estudiados (2006-2010) y la prueba de Matemáticas (PSUM) presenta una elevada capacidad predictiva comparable a las del NEM en contraste con la prueba de Lenguaje (PSUL), que muestra una capacidad predictiva baja al igual que la prueba de Ciencias Sociales (PSUH) y la prueba de Ciencias (PSUC) (Manzi et al., 2010). Esta comprobación es compartida por otros estudios respecto a la poca mejora de la capacidad predictiva de la PSU en comparación con la PAA, a pesar del aumento en sus contenidos y el perjuicio generado en los grupos de estudiantes de enseñanza media más vulnerables (Contreras, Corbalán y Redondo, 2007; Koljatic y Silva, 2010).

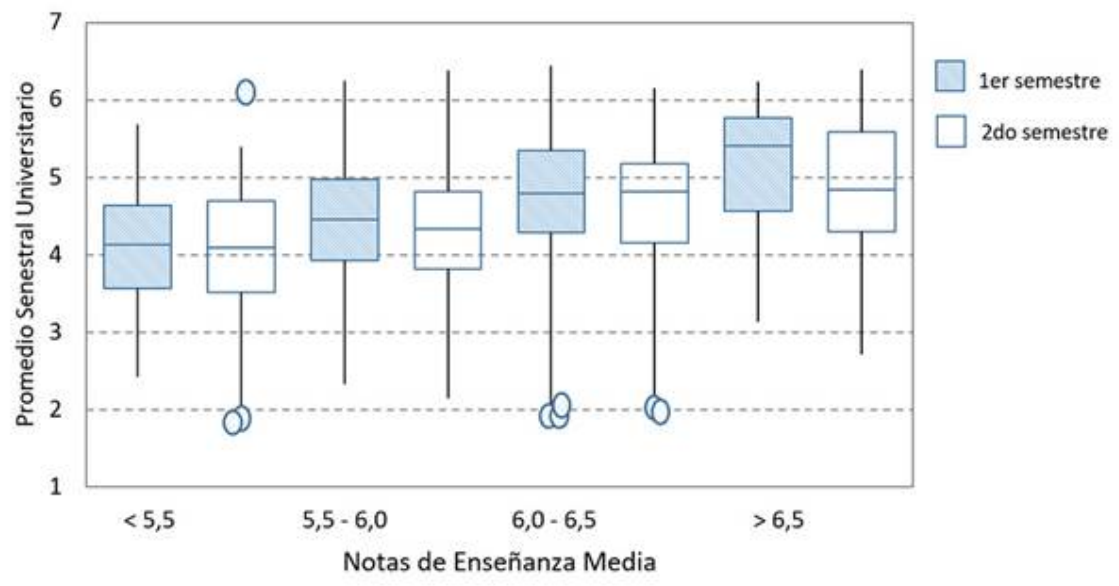

FIGURA 1

Relación NEM y promedio semestral ponderado de universidad, para todos los estudiantes. Elaboración propia.

De acuerdo con un estudio que analizó el método de selección y resultados académicos de la Escuela de Ingeniería de la Universidad de Chile, a partir de 3500 estudiantes que rindieron la antigua PAA -con ingreso entre 1994 y 2001-, se ha demostrado que, efectivamente, a mayor puntaje NEM, PEM y la prueba específica de Física (PEF), se comprobó una menor tasa de repetición y mejores promedios de notas (Fisher y Reppeto 2003).

Otros estudios coicidentes con nuestros resultados radican en el ámbito de las ciencias de la salud, donde se ha constatado que las NEM son un buen predictor del rendimiento académico en gran parte de las asignaturas de ciencias que se cursan en el primer año, en estas es casi nulo el valor predictivo de la PSU. En otras áreas científicas de la carrera, el comportamiento de la PSU matemática y ciencias, se alterna con la NEM en el valor predictivo del rendimiento académico (Rocha et al., 2009). 
$\mathrm{Al}$ realizar el análisis por género, para el caso femenino, se observa que el rendimiento académico del primer semestre de universidad se relaciona positivamente en mayor grado y de manera significativa con el puntaje de la PSU de Matemáticas $(\mathrm{r}=0,423 ; \mathrm{p}<0,01)$, seguida en un grado menor por el promedio de NEM $(\mathrm{r}=0,387 ; \mathrm{p}<0,01)$. El segundo semestre de rendimiento universitario, se relaciona con mayor fuerza y de manera significativa, con NEM $(r=0,407 ; \mathrm{p}<0,01)$, con una relación menor y significativa para los puntajes de PSU en Lenguaje, Matemáticas; sin embargo, para Historia y Ciencias la relación es baja y no significativa (tabla 4, figura 2,3).

TABLA 4

Efecto del rendimiento académico en la enseñanza media y factores de ingreso de mujeres, en relación al rendimiento del primer año académico universitario

\begin{tabular}{llll}
\hline & Promedios & $\begin{array}{l}\text { Promedio primer } \\
\text { semestre }\end{array}$ & $\begin{array}{l}\text { Promedio segundo } \\
\text { semestre }\end{array}$ \\
\hline Promedio notas NEM & 6,00 & $0,39\left(^{* *}\right)$ & $0,41\left(^{* *}\right)$ \\
\hline Puntaje PSU Lenguaje & 566,08 & $0,33(* *)$ & $0,26\left(^{* *}\right)$ \\
\hline Puntaje PSU Matemáticas & 583,31 & $0,43\left({ }^{* *}\right)$ & $0,31\left(^{* *}\right)$ \\
\hline Puntaje PSU Historia & 559,25 & $0,23\left({ }^{* *}\right)$ & 0,15 \\
\hline Puntaje PSU Ciencias & 527,01 & $0,33(* *)$ & 0,08 \\
\hline${ }^{*} \mathrm{p}<0,05 ;{ }^{* *} \mathrm{p}<0,01$ & & &
\end{tabular}

Otro aspecto social importante en el cual la PSU presenta un sesgo leve, pero apreciable, es en el desempeño de la PSUM, donde se puede apreciar una discriminación positiva hacia los hombres. Sin embargo, al considerar las NEM, salen favorecidas las mujeres. Si se combinan los resultados de ambas pruebas, se produce una compensación de las discriminaciones (Manzi et al., 2010). Desde el punto de vista del área de aprendizaje, estos resultados sugieren ahondar en el origen del comportamiento diferente según sexo, o tipo de establecimiento educacional, que podría llevar hasta los procesos de enseñanza-aprendizaje respectivos y su efecto en estos dos factores de predicción sobre desempeño académico en el primer año universitario de estudiantes de educación media (Manzi et al., 2010).

$\mathrm{Al}$ analizar el caso masculino, se observa que el rendimiento académico del primer semestre de universidad se relaciona positivamente en mayor grado y de manera significativa con el puntaje de la PSU de Matemáticas $(\mathrm{r}=0,341 ; \mathrm{p}<0,01)$, seguida en un grado menor por la PSU de Ciencias $(r=0,294 ; \mathrm{p}<0,01)$ y en tercer lugar el promedio de NEM ( $r=0,267, p<0,01)$. Sin embargo, el segundo semestre de rendimiento universitario, se relaciona con mayor fuerza y de manera significativa, con las notas de enseñanza media $(r=0,278 ; p<0,01)$, con una relación menor y significativa para los puntajes de PSU en Ciencias, Lenguaje. Por otra parte, existe una baja relación y no significativa para Matemáticas e Historia (tabla 5, figura 2, 3).

TABLA 5

Efecto del rendimiento académico en la enseñanza media y factores de ingreso de hombres, en relación al rendimiento del primer año académico universitario

\begin{tabular}{lccc}
\hline & Promedio & $\begin{array}{c}\text { Promedio primer } \\
\text { semestre }\end{array}$ & $\begin{array}{c}\text { Promedio segundo seme- } \\
\text { stre }\end{array}$ \\
\hline Promedio de notas NEM & 5,83 & $0,27\left(^{* *}\right)$ & $0,28\left(^{* \star}\right)$ \\
\hline Puntaje PSU Lenguaje & 580,25 & $0,25\left(^{(*)}\right)$ & $0,15\left({ }^{*}\right)$ \\
\hline Puntaje PSU Matemáticas & 603,40 & $0,34\left(^{(* *}\right)$ & 0,11 \\
\hline Puntaje PSU Historia & 599,15 & $0,15\left(^{*}\right)$ & 0,06 \\
\hline Puntaje PSU Ciencias & 542,49 & $0,29\left(^{* *}\right)$ & $0,23\left(^{* *}\right)$ \\
\hline${ }^{*} \mathrm{p}<0,05 ;{ }^{* *} \mathrm{p}<0,01$ & & & \\
\hline
\end{tabular}




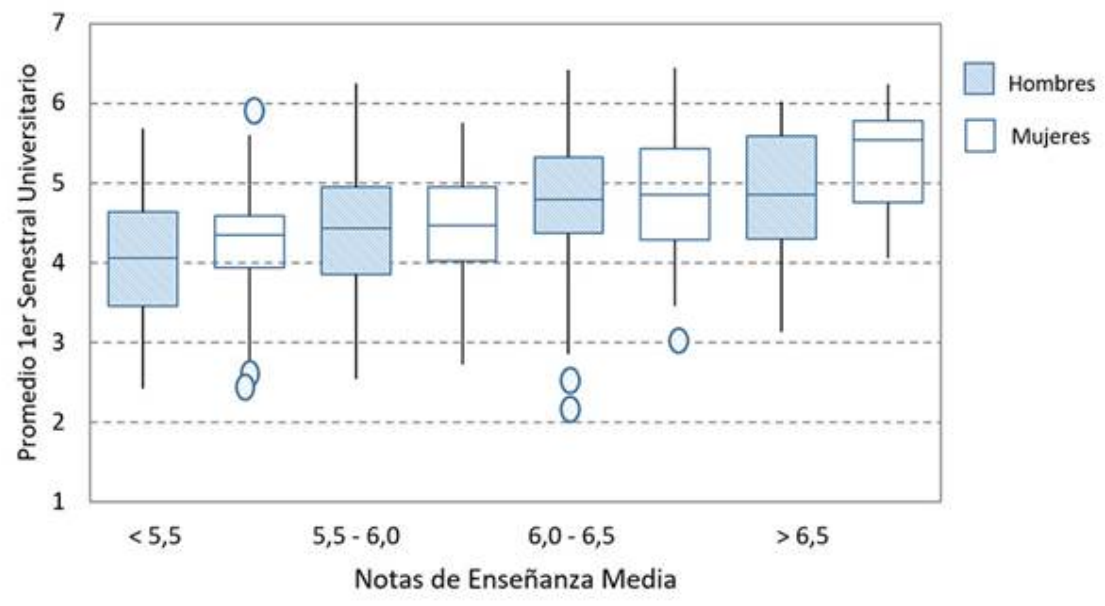

FIGURA 2

Relación NEM y Promedio Ponderado 1er Semestre en la universidad, para estudiantes mujeres y hombres Elaboración propia.

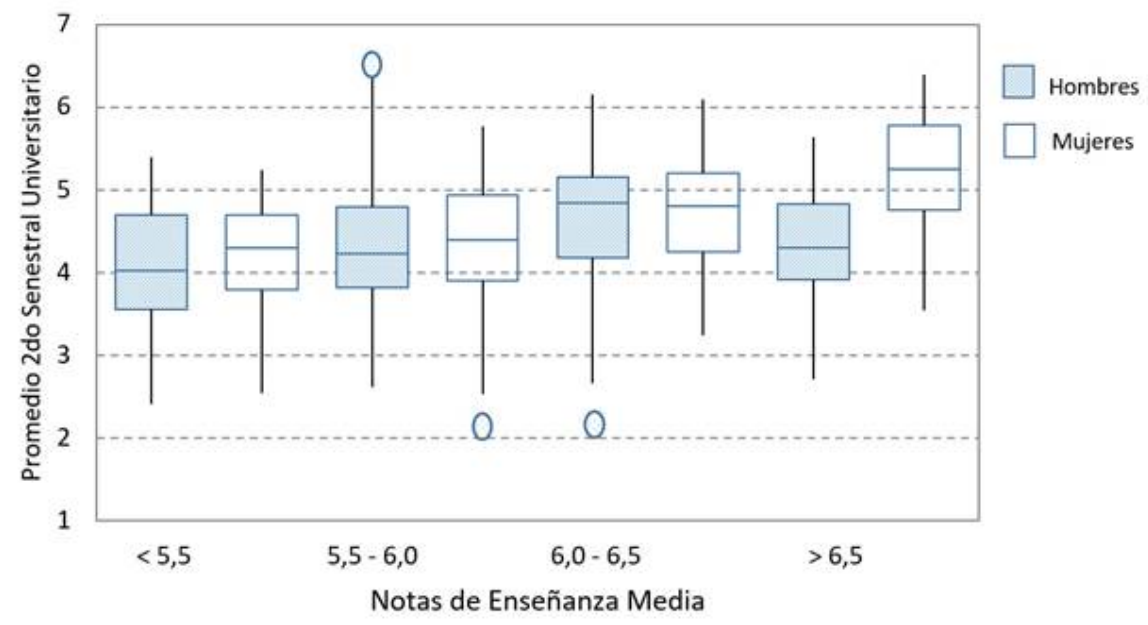

FIGURA 3

Relación NEM y Promedio Ponderado 2do Semestre en la universidad, para estudiantes mujeres y hombres Elaboración propia.

Las mujeres mantienen la misma tendencia que los hombres, pero presentando una correlación más alta entre promedios NEM y rendimiento académico universitarios. Por otro lado, sí mantienen una relación positiva y significativa entre la PSU de matemáticas y el rendimiento académico del primer y segundo semestre, contrastando con lo que sucede para los hombres con este factor de ingreso, en el cual desaparece su relación y significancia con el rendimiento del segundo semestre de universidad.

Respecto a indicadores adicionales para predecir el rendimiento en la universidad, los hallazgos de Pearson (2013) indican que el ranqueo de notas de enseñanza media tiene una mejor capacidad predictiva en el desempeño universitario de estudiantes que el puntaje PSU.

Otro estudio que ratifica nuestros hallazgos se relaciona con lo realizado por Meneses y Toro (2012), los cuales, en busca de mejorar la capacidad explicativa de las NEM en el desempeño universitario en la carrera de Derecho de la Universidad de Chile, incorporaron el ranqueo de estudiantes en la enseñanza media, lo que generó un incremento en medio punto porcentual de las respectivas notas, y ayudó a identificar mejor las capacidades académicas estudiantiles. 
La legislación chilena actual asigna parte del financiamiento universitario con base en los 27.500 mejores puntajes PSU, a pesar de su menor capacidad predictiva del rendimiento académico respecto a la NEM, de acuerdo con nuestros resultados. Meneses, Parra y Zenteno (2005) postularon que se puede agregar las NEM y el ranqueo de notas de enseñanza sin afectar significativamente el financiamiento, pero mejorando en forma notoria la capacidad predictiva del rendimiento en la universidad con las notas en enseñanza media.

Según otro estudio más reciente realizado con las admisiones 2003 - 2006, por Manzi et al. (2008), quienes utilizaron datos de 181.441 estudiantes de 25 universidades entre los años anteriormente mencionados, se establece -en cuanto al rendimiento (promedio ponderado acumulado, PPA) de estudiantes "que las notas de enseñanza media mantienen una capacidad predictiva equivalente y comparativamente alta para todos los años (r2003=0,23; r2004=0,25; r2005=0,23; r2006=0,23)" (p. 27).

Finalmente, es necesario destacar otros estudios que avalan nuestros resultados, relacionados con simulaciones realizadas por Larroucau et al. (2013), donde se le asigna al ranking de las notas de enseñanza media el 20\% de ponderación y se compensa con una baja equivalente de la ponderación de las PSU: los resultados obtenidos indican que la capacidad predictiva del comportamiento en la universidad mejora moderadamente.

\section{Conclusiones}

Los resultados de la investigación dejan en evidencia que los mejores predictores del rendimiento académico universitario para el primer año corresponden a las NEM. Se evidencia que su correlación es más fuerte, incluso al avanzar al segundo semestre académico.

Como predictores del rendimiento académico, los puntajes de las diferentes PSU tienen una relación inferior a la NEM, situación que, además, disminuye con el avance al segundo semestre académico. Sin embargo, la PSUM en el primer semestre tiene una relación significativa con el rendimiento académico, la que pierde fuerza el segundo semestre.

Es necesario que la carrera de Ingeniería Comercial revise las ponderaciones de las diferentes pruebas de ingreso, asignando un mayor peso a las NEM, a continuación la PSUM y finalmente la PSU global.

La asignación de financiamiento estatal a las universidades del CRUCh, que actualmente se realiza con base en la PSU, debiera incorporar como factor preponderante las NEM, con la justificación de que ha demostrado ser un mejor predictor del desempeño académico del estudiantado universitario.

\section{REFERENCIAS}

Bravo D., Del Pino, G., Donoso, G., Manzi, J., Martínez, M., y Pizarro, R. (2010). Resultados de la aplicación de pruebas de selección universitaria. Admisión 2006-2010. Comité Técnico Asesor del CRUCH. Recuperado de http://sistemadeadmision.consejoderectores.cl/public/pdf/publicaciones/ Resultados_de_la_Aplicacion_Admisiones_2006-2010.pdf

Contreras, M., Corbalán, F., y Redondo, J. (2007). Cuando la suerte está echada: Estudio cuantitativo de los factores asociados al rendimiento en la PSU. REICE: Revista Electrónica Iberoamericana sobre Calidad, Eficacia y Cambio en Educación. 5(5), 259-263. Recuperado de http://dialnet.unirioja.es/servlet/articulo? codigo $=2521630$

Departamento de Evaluación, Medición y Registro Educacional (DEMRE), Universidad de Chile. (2013). Puntaje Ranqueo. Recuperado de http://www.demre.cl/proceso-admision/factores-seleccion/puntaje-ranqueo

Edwards D., Coates, H. y Friedman, T. (2012). A survey of international practice in university admissions testing. Higher Education Management and Policy, 24(1), 1-18. doi: http://dx.doi.org/10.1787/ hemp-24-5k9bdck3bkr8 
Fisher, R. y Reppeto, A. (2003). Método de selección y resultados académicos: Escuela de Ingeniería, Universidad de Chile. Recuperado de http://www.cepchile.cl/dms/lang_1/doc_3234.html

Gil, F., Paredes, R. y Sánchez, I. (2013). El ranqueo de las notas: Inclusión con excelencia. Centro de Políticas Públicas UC, 8(60), 3-19. Recuperado de http://politicaspublicas.uc.cl/wp-content/uploads/2015/02/serie-no-60-elranking-de-las-notas-inclusion-con-excelencia.pdf

Giusti, A. (2004). Evaluación de predictores de rendimiento académico en la carrera de Ingeniería Forestal de la Universidad Austral de Chile (Tesis de pregrado). Universidad Austral de Chile. Recuperado de http:// cybertesis.uach.cl/tesis/uach/2004/fifg538e/doc/fifg538e.pdf

Koljatic, M. y Silva, M. (2013). Opening a side-gate: engaging the excluded in Chilean higher education through testblind admission. Studies in Higher Education, 38(10), 1427-1441. doi: 10.1080/03075079.2011.623299

Koljatic, M. y Silva, M. (2010). Algunas reflexiones a siete años de la implementación de la PSU. Estudios Públicos, 120, 125-146. Recuperado de http://dialnet.unirioja.es/servlet/articulo?codigo=3705815

Larroucau, T., Ríos, I. y Mizala, A. (2013). Efecto de la incorporación del ranking de notas en el proceso de admisión a las universidades chilenas. Pensamiento Educativo, 52(2), 95-118. http://dx.doi.org/10.7764/PEL.52.1.2015.8

Manzi, J., Bosch, A., Bravo, D., Pino, G., Donoso, G. y Pizarro, R. (2010). Validez diferencial y sesgo en la predictividad de las pruebas de admisión a las universidades chilenas (PSU). Rev. Iber. Eval. Educ., 3(2), 29-48. Recuperado de http://www.rinace.net/riee/numeros/vol3-num2/art2.pdf

Manzi, J., Bravo, D., Del Pino, G., Donoso, G., Martínez, M. y Pizarro, R. (2008). Estudio acerca de la validez predictiva de los factores de selección a las universidades del Consejo de Rectores. Comité Técnico Asesor del CRUCH. Recuperado dehttp://sistemadeadmision.consejoderectores.cl/public/pdf/publicaciones/ Estudio_de_Validez_Predictiva_2003-2006.pdf

Meneses, F. y Toro, J. (2012). Predicción de notas en Derecho de la Universidad de Chile: ¿Sirve el ranqueo? ISEES 10, 43-58. Recuperado de http://dialnet.unirioja.es/descarga/articulo/4420036.pdf

Meneses, F., Parra, A. y Zenteno, L. (2005). Can the Chilean university entrance systems be improved? The use of High School ranqueo in the Universidad de Chile, Universidad Católica de Chile and Universidad de Santiago. MPRA Paper 23048, 1-8. Recuperado de https://mpra.ub.uni-muenchen.de/23048/1/ MPRA_paper_23048.pdf

PEARSON. (2013). Informe final evaluación de la PSU Chile. Recuperado de http://www.mineduc.cl/usuarios/ mineduc/doc/201301311058200.ChilePSU-Resumen_Ejecutivo.pdf

Rocha, F., Acebedo, C. y Flores, M. (2009). Perfil de ingreso de los estudiantes de la Carrera de Tecnología Médica de la Universidad de Concepción (cohortes 2006 al 2008). Determinación de variables predictivas del rendimiento académico en asignaturas de ciencia. Revista Educación Ciencias de la Salud, 6(1), 28-33. Recuperado de http:// www2.udec.cl/ofem/recs/anteriores/vol612009/artrev6109a.pdf\#page $=24$

\section{Notas}

[3] Los tipos de establecimientos educacionales a nivel de Enseñanza Media y reconocidos por el Estado de Chile, corresponden a: Municipales (Establecimientos públicos de propiedad y financiamiento principalmente estatal, administrados por las municipalidades del país), Particulares subvencionados (Establecimientos de propiedad y administración privada, pero reciben financiamiento estatal mediante subvención por alumno matriculado y efectivamente asistiendo a clases) y Particulares pagados (Establecimientos privados propiamente tales, cuya propiedad, administración y financiamiento corresponde a particulares y a las familias del alumnado).

\section{BY-NC-ND}

